\title{
The association of serum long-chain $n-3$ PUFA and hair mercury with exercise cardiac power in men
}

\author{
Behnam Tajik, Sudhir Kurl, Tomi-Pekka Tuomainen and Jyrki K. Virtanen* \\ Kuopio Campus, Institute of Public Health and Clinical Nutrition, University of Eastern Finland, 70211 Kuopio, Finland
}

(Submitted 5 February 2016 - Final revision received 8 April 2016 - Accepted 27 April 2016 - First published online 3 June 2016)

\begin{abstract}
Long-chain $n$-3 PUFA from fish and exercise capacity are associated with CVD risk. Fish, especially large and old predatory fish, may contain $\mathrm{Hg}$, which may attenuate the inverse association of long-chain $n-3$ PUFA with CVD. However, the associations of long-chain $n-3$ PUFA or Hg exposure with exercise capacity are not well known. We aimed to evaluate the associations of serum long-chain $n$-3 PUFA EPA, docosapentaenoic acid (DPA) and DHA and hair $\mathrm{Hg}$ with exercise cardiac power (ECP, a ratio of $\mathrm{VO}_{2 \text { max }}$ maximal systolic blood pressure (SBP) during an exercise test), a measure for exercise capacity. For this, data from the population-based Kuopio Ischaemic Heart Disease Risk Factor Study were analysed cross-sectionally in order to determine the associations between serum long-chain $n-3$ PUFA, hair Hg and ECP in 1672 men without CVD, aged 42-60 years. After multivariate adjustments, serum total long-chain $n$-3 PUFA concentration was associated with higher ECP and $\mathrm{VO}_{2 \max }\left(P_{\text {trend }}\right.$ across quartiles $=0.04$ and $P_{\text {trend }}=0.02$, respectively $)$, but not with maximal SBP $\left(P_{\text {trend }}=0.69\right)$. Associations were generally similar when EPA, DPA and DHA were evaluated individually. Hair $\mathrm{Hg}$ was not associated with $\mathrm{ECP}, \mathrm{VO}_{2 \mathrm{max}}$ or maximal SBP. However, the associations of total long-chain $n-3$ PUFA $\left(P_{\text {interaction }}=0.03\right)$ and EPA $\left(P_{\text {interaction }}=0.02\right)$ with higher $\mathrm{VO}_{2 \text { max }}$ were stronger among men with lower hair Hg. Higher serum long-chain $n$-3 PUFA concentration, mainly a marker for fish consumption in this study population, was associated with higher ECP and $\mathrm{VO}_{2 \max }$ in middle-aged men from eastern Finland.
\end{abstract}

\section{Key words: Fatty acids: Exercise capacity: Cohort studies: Cross-sectional study}

Low exercise capacity during an exercise test has been established as an independent predictor of risk for total mortality and $\mathrm{CVD}^{(1,2)}$. Exercise cardiac power (ECP), which is defined as a ratio of directly measured $\mathrm{VO}_{2 \text { max }}$ :peak systolic blood pressure (SBP) during exercise test, is an accurate measure for exercise capacity and it is known to be an independent predictor of $\mathrm{CVD}^{(3)}$. The advantage of ECP compared with other exercise capacity measurements is that ECP provides information not only about cardiorespiratory fitness but also considers the differences in cardiovascular resistance and cardiac afterload ${ }^{(3,4)}$.

Although little is known about ECP and risk of CVD, previously in the Kuopio Ischaemic Heart Disease Risk Factor (KIHD) study cohort, lower ECP was associated with increased risk of sudden cardiac death and stroke in men ${ }^{(3,4)}$. In addition, low cardiorespiratory fitness $\left(\mathrm{VO}_{2 \max }\right)$ and increased SBP during exercise were associated with higher risk of cardiovascular events and CVD-related mortality in the KIHD cohort $^{(5-8)}$.

Substantial evidence from epidemiological studies, including KIHD study, demonstrates that long-chain $n$-3 PUFA may reduce the risk of $\mathrm{CVD}^{(9-11)}$. To the best of our knowledge, no previous studies have been conducted to evaluate the association of these fatty acids with ECP. However, a few small supplementation studies have assessed the efficacy of long-chain $n-3$ PUFA on $\mathrm{VO}_{2 \max }{ }^{(12-20)}$ and SBP during exercise ${ }^{(19,21,22)}$, but the findings are inconsistent.

We evaluated the association of serum long-chain $n$-3 PUFA concentrations with ECP, $\mathrm{VO}_{2 \max }$ and maximal SBP during an exercise test among middle-aged and older men from the KIHD cohort. We also evaluated whether high hair $\mathrm{Hg}$ concentration, a biomarker for long-term $\mathrm{Hg}$ exposure ${ }^{(23)}$, is associated with ECP and whether it could modify the associations with long-chain $n$-3 PUFA, as it has been shown to do with the risk of CVD in the KIHD population ${ }^{(10,11)}$.

\section{Methods}

\section{Study population}

Subjects were participants of the KIHD, which is a prospective, population-based study designed to investigate risk factors for CVD, carotid atherosclerosis and related outcomes in a randomly selected sample of men from eastern Finland ${ }^{(24)}$. The baseline examinations were carried out in 1984-1989. Of the 3235 eligible men aged 42, 48, 54 or 60 years who lived in

Abbreviations: DPA, docosapentaenoic acid; ECP, exercise cardiac power; KIHD, Kuopio Ischaemic Heart Disease Risk Factor Study; SBP, systolic blood pressure.

* Corresponding author: J. K. Virtanen, fax +358 17162 936, email jyrki.virtanen@uef.fi 
the city of Kuopio or its surrounding areas, 2682 men (82.9\%) were recruited to the baseline study. The baseline characteristics of the entire study population have been described previously ${ }^{(24)}$. The KIHD study protocol was approved by the Research Ethics Committee of the University of Kuopio. All subjects gave their written informed consent for participation. From the analyses, we excluded participants with missing data on ECP measurements ( $n$ 207), a history of CVD ( $n$ 677) or those with missing data on serum long-chain $n$-3 PUFA ( $n$ 113) or hair $\mathrm{Hg}(n$ 13). After exclusions, 1672 men were included in the final analysis.

\section{Measurements}

Subjects provided their hair and venous blood samples between 08.00 and 10.00 hours at baseline. Repeat hair samples were collected from twenty-one subjects 4-9 years (mean, 6 years) after baseline examinations to survey the tracking of hair $\mathrm{Hg}$ values over time. The subjects were instructed to abstain from alcohol for $3 \mathrm{~d}$ and from smoking and eating for $12 \mathrm{~h}$ before providing samples. Comprehensive description of the determination of serum lipids and lipoproteins ${ }^{(25)}$, assessment of medical history and use of medications ${ }^{(25)}$, smoking status ${ }^{(25)}$, alcohol consumption $^{(25)}$, resting blood pressure ${ }^{(25)}$ and physical activity ${ }^{(26)}$ have been reported previously. Hypertension diagnosis was defined as SBP/diastolic blood pressure $>140 / 90 \mathrm{mmHg}$ at study visit, clinical diagnosis of hypertension or use of hypertensive medication. Serum C-reactive protein (CRP) was measured using an immunometric assay (Immulite High Sensitivity CRP Assay; DPC). Dietary intakes were assessed using 4-d food recording at the time of blood sampling ${ }^{(27)}$. Educational status was assessed in years using self-administered questionnaires ${ }^{(27)}$.

\section{Serum fatty acid and mercury measurements}

Serum fatty acids were determined in a single gas chromatographic run without pre-separation as described previously ${ }^{(28)}$. Serum fatty acids were extracted using chloroform-methanol solution. The chloroform phase was evaporated and treated with sodium methoxide, which methylated esterified fatty acids. Quantification was carried out with reference standards purchased from Nu-Check Prep Inc. Each analyte had an individual reference standard, and the internal standard was eicosane. Fatty acids were chromatographed in an NB-351 capillary column (HNU-Nordion) by a Hewlett-Packard 5890 Series II gas chromatograph with a flame ionisation detector (Hewlett-Packard Company, since 1999 Agilent Technologies Inc.). Results for fatty acids were obtained in $\mu \mathrm{mol} / \mathrm{l}$, and in the data analyses proportion of fatty acids from total fatty acids was used. The CV\% was 9.4\% for EPA (20:5n-3), $12.7 \%$ for docosapentaenoic acid (DPA, $22: 5 n-3$ ) and $11.9 \%$ for DHA (22:5n-3). For the serum total long-chain $n-3$ PUFA, we used the sum of EPA, DPA and DHA.

Hair Hg was detected by flow injection analysis-cold vapour atomic absorption spectrometry and amalgamation ${ }^{(29)}$. The Pearson's correlation coefficient between the original and the repeat measurement collected after 4-9 years was 0.91.

\section{Assessment of exercise cardiac power}

A maximal symptom-limited exercise tolerance test was performed between 08.00 and 10.00 hours using an electrically braked cycle ergometer (Medical Fitness Equipment 400 L bicycle ergometer) ${ }^{(30)}$. The standardised testing protocol comprised of an increase in the workload of $20 \mathrm{~W} / \mathrm{min}$ with the direct analyses of respiratory gases (Medical Graphics). ECP was measured by the ratio of measured $\mathrm{VO}_{2 \max }$ :peak $\mathrm{SBP}^{(3)}$. $\mathrm{VO}_{2 \max }$ was defined as the highest value for or the plateau on $\mathrm{VO}_{2}$. Blood pressure was measured every $2 \mathrm{~min}$ both manually and automatically during exercise until the test was stopped and every 2 min after exercise. In the present study, we used only manually measured blood pressure values. The highest SBP achieved during the exercise test was defined as the maximum exercise SBP. For safety reasons, all tests were supervised by an experienced physician with assistance from an experienced nurse. Electrocardiography was recorded continuously with the Kone 620 electrocardiograph (Kone) ${ }^{(7,8)}$.

\section{Statistical analysis}

The univariate associations between serum EPA + DPA+DHA concentrations and demographic, lifestyle and clinical characteristics at baseline were assessed by means and linear regressions for continuous variables and by the $\chi^{2}$-test for categorical variables. Correlations between individual longchain $n-3$ PUFA were evaluated by calculating Spearman's correlation coefficients. Linear regression models were used to determine the association of serum long-chain $n-3$ PUFA with $\mathrm{ECP}, \mathrm{VO}_{2 \max }$ and maximum SBP during exercise. The mean values of $\mathrm{ECP}, \mathrm{VO}_{2 \max }$ and maximum SBP during exercise in the exposure quartiles were analysed using ANCOVA.

In addition, two models were run to adjust for potential cofounders. Model 1 was adjusted for age (years) and examination year, and model 2 included the variables in the model $1+\mathrm{BMI}$ $\left(\mathrm{kg} / \mathrm{m}^{2}\right.$ ), smoking status (non-smoker, previous smoker, current smoker $<20$ cigarettes/d and $\geq 20$ cigarettes per $\mathrm{d}$ ), leisure-time physical activity $(\mathrm{kJ} / \mathrm{d}(\mathrm{kcal} / \mathrm{d}))$, use of drugs for hypertension (yes/no), bronchial asthma (yes/no), LDL-cholesterol and HDLcholesterol (mmol/l), CRP levels (mg/l) and intakes of energy $(\mathrm{kJ} / \mathrm{d}$ (kcal/d)), carbohydrates (g/d) and alcohol (g/week). The cohort mean was used to replace missing values in covariates $(<0.5 \%)$. Test for linear trend across quartiles was assessed using the median value in each quartile as the continuous variable in the linear regression model. Statistical significance of the interactions on a multiplicative scale was assessed by stratified analysis with hair $\mathrm{Hg}$ divided by the median and likelihood ratio tests with a crossproduct term. For assessing the clinical significance, we calculated effect sizes based on Cohen's $d$ index (the difference between the group means divided by the standard deviation of the comparison

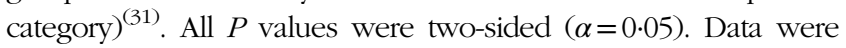
analysed using SPSS software version 21 for windows (IBM Corp.).

\section{Results}

\section{Baseline characteristics}

Baseline characteristics of the participants are presented in Table 1. Men with higher serum total long-chain n-3 PUFA 
Table 1. Baseline characteristics according to total serum long-chain $n-3$ PUFA

(Mean values and standard deviations; percentages)

\begin{tabular}{|c|c|c|c|c|c|c|c|c|c|}
\hline \multirow[b]{3}{*}{ Variables } & \multicolumn{8}{|c|}{ Serum total $n$-3 PUFA quartile } & \multirow[b]{3}{*}{$P_{\text {for trend }}{ }^{\star}$} \\
\hline & \multicolumn{2}{|c|}{ Q1 $(<3.67)(n 418)$} & \multicolumn{2}{|c|}{ Q2 (3.67-4.38) (n 418) } & \multicolumn{2}{|c|}{ Q3 (4.39-5.40) (n 418) } & \multicolumn{2}{|c|}{ Q4 (>5.40) (n 418) } & \\
\hline & Mean & $\mathrm{SD}$ & Mean & $\mathrm{SD}$ & Mean & $\mathrm{SD}$ & Mean & $\mathrm{SD}$ & \\
\hline Age (years) & $52 \cdot 0$ & $5 \cdot 3$ & $52 \cdot 1$ & $5 \cdot 2$ & $52 \cdot 4$ & $5 \cdot 3$ & $52 \cdot 7$ & $5 \cdot 1$ & 0.05 \\
\hline Education (years) & 8.9 & $3 \cdot 3$ & $8 \cdot 7$ & $3 \cdot 4$ & $8 \cdot 8$ & 3.6 & 9.6 & $4 \cdot 0$ & 0.001 \\
\hline BMI $\left(\mathrm{kg} / \mathrm{m}^{2}\right)$ & $26 \cdot 6$ & $3 \cdot 4$ & $26 \cdot 5$ & $3 \cdot 1$ & $26 \cdot 9$ & 3.6 & $27 \cdot 0$ & 3.4 & 0.05 \\
\hline Current smoker (\%) & \multicolumn{2}{|c|}{$31 \cdot 1$} & \multicolumn{2}{|c|}{28.9} & \multicolumn{2}{|c|}{$30 \cdot 4$} & \multicolumn{2}{|c|}{27.5} & 0.32 \\
\hline Leisure-time physical activity (kJ/d) & 556 & 694 & 548 & 669 & 556 & 594 & 653 & 858 & 0.03 \\
\hline Leisure-time physical activity (kcal/d) & 133 & 166 & 131 & 160 & 133 & 142 & 156 & 205 & 0.03 \\
\hline C-Reactive protein $(\mathrm{mg} / \mathrm{l})$ & $2 \cdot 27$ & 3.96 & 2.00 & 3.03 & $2 \cdot 28$ & 3.61 & 1.91 & 3.08 & 0.23 \\
\hline Serum TAG $(\mathrm{mmol} / \mathrm{l})$ & 1.52 & 0.93 & $1 \cdot 26$ & 0.80 & $1 \cdot 16$ & 0.57 & 1.03 & 0.51 & $<0.001$ \\
\hline Serum HDL-cholesterol (mmol/l) & $1 \cdot 22$ & 0.28 & 1.29 & 0.28 & $1 \cdot 33$ & 0.28 & 1.35 & 0.31 & $<0.001$ \\
\hline Serum LDL-cholesterol (mmol/l/) & 3.82 & 0.92 & 3.99 & 0.97 & 4.08 & 1.02 & $4 \cdot 12$ & 0.96 & $<0.001$ \\
\hline Blood glucose $(\mathrm{mmol} / \mathrm{l})$ & $4 \cdot 72$ & 0.96 & $4 \cdot 36$ & 0.84 & $4 \cdot 73$ & 0.82 & $4 \cdot 73$ & 0.90 & 0.40 \\
\hline Systolic blood pressure $(\mathrm{mmHg})$ & 136 & 17 & 134 & 15 & 134 & 16 & 134 & 17 & 0.140 \\
\hline Diastolic blood pressure $(\mathrm{mmHg})$ & 90 & 11 & 89 & 10 & 89 & 10 & 89 & 11 & 0.180 \\
\hline Energy intake (kJ/d) & 10230 & 2807 & 10280 & 2494 & 9870 & 2594 & 9581 & 2506 & $<0.001$ \\
\hline Energy intake (kcal/d) & 2445 & 671 & 2457 & 596 & 2359 & 620 & 2290 & 599 & $<0.001$ \\
\hline Carbohydrate intake (g/d) & 258 & 38 & 254 & 38 & 246 & 39 & 245 & 36 & $<0.001$ \\
\hline Alcohol intake (g/week) & 57 & 92 & 66 & 103 & 82 & 125 & 85 & 116 & $<0.001$ \\
\hline Diabetes (\%) & \multicolumn{2}{|c|}{5.5} & \multicolumn{2}{|c|}{2.9} & \multicolumn{2}{|c|}{$4 \cdot 3$} & \multicolumn{2}{|c|}{$4 \cdot 8$} & 0.99 \\
\hline Hypertension (\%) & \multicolumn{2}{|c|}{$59 \cdot 3$} & \multicolumn{2}{|c|}{$53 \cdot 8$} & \multicolumn{2}{|c|}{$56 \cdot 5$} & \multicolumn{2}{|c|}{$53 \cdot 1$} & 0.140 \\
\hline Drug for hypertension (\%) & \multicolumn{2}{|c|}{$19 \cdot 4$} & \multicolumn{2}{|c|}{$12 \cdot 4$} & \multicolumn{2}{|c|}{$12 \cdot 4$} & \multicolumn{2}{|c|}{$11 \cdot 7$} & 0.006 \\
\hline Serum EPA (\%)† & 0.97 & 0.25 & 1.29 & 0.24 & $1 \cdot 71$ & 0.30 & $2 \cdot 80$ & $1 \cdot 12$ & $<0.001$ \\
\hline Serum DPA (\%)† & 0.47 & 0.07 & 0.53 & 0.07 & 0.56 & 0.78 & 0.64 & $0 \cdot 10$ & $<0.001$ \\
\hline Serum DHA (\%)† & 1.74 & 0.28 & $2 \cdot 20$ & 0.26 & 2.57 & 0.29 & $3 \cdot 39$ & 0.66 & $<0.001$ \\
\hline Hair Hg $(\mu \mathrm{g} / \mathrm{g})$ & $1 \cdot 18$ & $1 \cdot 30$ & 1.65 & $1 \cdot 78$ & $2 \cdot 20$ & 2.04 & $2 \cdot 71$ & $2 \cdot 34$ & $<0.001$ \\
\hline
\end{tabular}

Q, quartiles; DPA, docosapentaenoic acid.

* Participant characteristics at baseline were assessed by means and linear regressions for continuous variables and the $\chi^{2}$-test for categorical variables.

$\dagger$ Proportion of all serum fatty acids.

concentrations were more likely to be older $(P=0.05)$ and have higher education $(P=0 \cdot 001)$, BMI $(P=0 \cdot 05)$, leisure-time physical activity $(P=0 \cdot 03)$, serum HDL- and LDL-cholesterol concentrations $(P<0.001)$, hair $\mathrm{Hg}$ concentration $(P=0.05)$ and alcohol intake $(P<0.001)$. They also had lower carbohydrate intake $(P<0 \cdot 001)$, lower total energy intake $(P<0 \cdot 001)$, lower serum TAG levels $(P<0 \cdot 001)$ and they were less likely to use anti-hypertensive drugs $(P=0 \cdot 001)$.

The mean serum concentrations, as a percentage of all serum fatty acids, were 4.72 (SD 1.60 ) \% for total long-chain n-3 PUFA, 1.69 (SD 0.92)\% for EPA, 0.55 (SD 0.10)\% for DPA and 2.48 ( $\mathrm{SD} 0.73$ ) $\%$ for DHA. The correlations between the individual long-chain $n$-3 PUFA were 0.70 for EPA and DHA, 0.56 for EPA and DPA and 0.41 for DHA and DPA. The mean hair content of $\mathrm{Hg}$ was $1.94 \mu \mathrm{g} / \mathrm{g}$ and ranged from 0 to $15 \cdot 67 \mu \mathrm{g} / \mathrm{g}$.

\section{Serum long-chain n-3 PUFA, hair mercury and exercise cardiac power}

The mean ECP was 12.46 (sD 3.07) ml/mmHg. After adjustment for age and examination year (model 1), higher serum total longchain $n$-3 PUFA concentration was associated with higher ECP (the mean difference between extreme quartiles was $0.42 \mathrm{ml} /$ mmHg (95\% CI 0.03, 0.81, $P_{\text {trend }}$ across quartiles =0.04)). Further multivariate adjustments had little impact on the association (model 2, Table 2). When the fatty acids were investigated individually, generally similar direct associations were observed with EPA, DPA and DHA (Table 2). The effect sizes, based on Cohen's $d$ index, were $0 \cdot 10$ for total serum long-chain $n$-3 PUFA, 0.05 for EPA, 0.15 for DPA, 0.20 for DHA and 0.21 for $\mathrm{Hg}$.

Hair Hg concentration was not associated with ECP (Table 2). Although we could not find statistically significant interactions between the long-chain $n-3$ PUFA and hair $\mathrm{Hg}$ for ECP ( $P_{\text {for interaction }}=0.15$ for the total long-chain $n-3$ PUFA, $P=0.08$ for EPA, $P=0.47$ for DPA and $P=0.50$ for DHA), we observed statistically significant associations between the long-chain $n-3$ PUFA and higher ECP only in participants with lower hair $\mathrm{Hg}$ content (<median 1.30 $\mu \mathrm{g} / \mathrm{g}$ ) (online Supplementary Table S1).

\section{Serum long-chain n-3 PUFA, hair mercury and $\mathrm{VO}_{2 \text { max }}$}

The mean $\mathrm{VO}_{2 \max }$ was 2545 (sD 559) $\mathrm{ml} / \mathrm{min}$. Higher serum total long-chain $n$-3 PUFA concentration was associated with higher $\mathrm{VO}_{2 \max }$ (Table 3). The extreme-quartile difference in the multivariate-adjusted model was $83 \mathrm{ml} / \mathrm{min}$ (95\% CI 15, 152, $P_{\text {trend }}$ across quartiles $\left.=0.02\right)$. The associations were again generally similar with EPA, DPA and DHA (Table 3). The effect sizes were 0.13 for total serum long-chain $n$-3 PUFA, 0.05 for EPA, 0.28 for DPA, $0 \cdot 15$ for DHA and $0 \cdot 19$ for $\mathrm{Hg}$.

We did not find a statistically significant association between hair $\mathrm{Hg}$ content and $\mathrm{VO}_{2 \max }$ (Table 3 ). However, adjusting for hair $\mathrm{Hg}$ content modestly attenuated the associations between the long-chain $n-3$ PUFA and $\mathrm{VO}_{2 \max }$ (Table 3 ). Furthermore, the associations were stronger among those with hair $\mathrm{Hg}$ below 
Table 2. Exercise cardiac power ( $\mathrm{ml} / \mathrm{mmHg}$ ) in quartiles of serum long-chain $n-3$ PUFA and hair mercury* (Mean values and $95 \%$ confidence intervals)

\begin{tabular}{|c|c|c|c|c|c|c|c|c|c|c|c|}
\hline & \multicolumn{8}{|c|}{ Exposure quartile } & \multirow[b]{3}{*}{$P_{\text {for trend }}$} & \multirow{2}{*}{\multicolumn{2}{|c|}{ Mean difference $†$}} \\
\hline & \multicolumn{2}{|c|}{$1(n 418)$} & \multicolumn{2}{|c|}{$2(n 418)$} & \multicolumn{2}{|r|}{$3(n 418)$} & \multicolumn{2}{|c|}{$4(n 418)$} & & & \\
\hline & Mean & $95 \% \mathrm{Cl}$ & Mean & $95 \% \mathrm{Cl}$ & Mean & $95 \% \mathrm{Cl}$ & Mean & $95 \% \mathrm{Cl}$ & & Mean & $95 \% \mathrm{Cl}$ \\
\hline $\mathrm{EPA}+\mathrm{DPA}+\mathrm{DHA}(\%)$ & \multicolumn{2}{|r|}{$<3.67$} & \multicolumn{2}{|c|}{$3 \cdot 67-4 \cdot 38$} & \multicolumn{2}{|r|}{$4 \cdot 39-5 \cdot 40$} & \multicolumn{2}{|r|}{$>5 \cdot 40$} & & & \\
\hline Model 1‡ & $12 \cdot 15$ & $11 \cdot 88,12 \cdot 43$ & 12.52 & $12 \cdot 25,12 \cdot 80$ & 12.59 & $12 \cdot 31,12 \cdot 86$ & 12.57 & $12 \cdot 30,12 \cdot 85$ & 0.04 & 0.42 & $0.03,0.81$ \\
\hline Model $2 \S$ & $12 \cdot 15$ & $11 \cdot 89,12.42$ & $12 \cdot 47$ & $12 \cdot 21,12 \cdot 72$ & $12 \cdot 65$ & $12 \cdot 39,12 \cdot 91$ & $12 \cdot 57$ & $12 \cdot 31,12 \cdot 83$ & 0.04 & 0.42 & $0.04,0.80$ \\
\hline Model $2+$ hair $\mathrm{Hg}$ & $12 \cdot 16$ & $11 \cdot 89,12 \cdot 43$ & 12.47 & $12 \cdot 21,12 \cdot 73$ & $12 \cdot 65$ & $12 \cdot 39,12 \cdot 91$ & 12.56 & $12 \cdot 30,12 \cdot 83$ & 0.06 & 0.40 & $0.01,0.80$ \\
\hline EPA $(\%)$ & \multicolumn{2}{|r|}{$<1 \cdot 13$} & \multicolumn{2}{|c|}{$1.13-1.49$} & \multicolumn{2}{|r|}{$1.50-2.01$} & \multicolumn{2}{|r|}{$>2.01$} & & & \\
\hline Model 1 & $12 \cdot 17$ & $11 \cdot 90,12 \cdot 45$ & $12 \cdot 43$ & $12 \cdot 15,12 \cdot 70$ & 12.75 & $12.47,13.02$ & 12.49 & $12 \cdot 21,12 \cdot 77$ & $0 \cdot 13$ & 0.32 & $-0.07,0.72$ \\
\hline Model 2 & $12 \cdot 12$ & $11 \cdot 86,12 \cdot 39$ & $12 \cdot 39$ & $12 \cdot 13,12 \cdot 65$ & $12 \cdot 76$ & $12 \cdot 50,13 \cdot 02$ & 12.56 & $12 \cdot 30,12 \cdot 83$ & 0.03 & 0.44 & $0.05,0.83$ \\
\hline Model $2+$ hair Hg & $12 \cdot 13$ & $11 \cdot 86,12 \cdot 40$ & $12 \cdot 40$ & $12 \cdot 14,12 \cdot 66$ & $12 \cdot 76$ & $12 \cdot 50,13 \cdot 01$ & 12.55 & $12 \cdot 28,12 \cdot 82$ & 0.05 & 0.42 & $0.03,0.82$ \\
\hline DPA (\%) & \multicolumn{2}{|r|}{$<0.48$} & \multicolumn{2}{|c|}{$0.48-0.54$} & \multicolumn{2}{|c|}{$0.55-0.62$} & \multicolumn{2}{|r|}{$>0.62$} & & & \\
\hline Model 1 & $12 \cdot 01$ & $11 \cdot 74,12 \cdot 29$ & $12 \cdot 51$ & $12 \cdot 39,12 \cdot 79$ & $12 \cdot 51$ & $12 \cdot 24,12 \cdot 78$ & $12 \cdot 80$ & $12.52,13.07$ & $<0.001$ & 0.79 & $0.40,1.17$ \\
\hline Model 2 & $12 \cdot 20$ & $11.93,12.46$ & 12.55 & $12 \cdot 29,12 \cdot 80$ & $12 \cdot 44$ & $12 \cdot 81,12 \cdot 70$ & $12 \cdot 65$ & $12.39,12.91$ & 0.04 & 0.46 & $0.08,0.84$ \\
\hline Model $2+$ hair $\mathrm{Hg}$ & $12 \cdot 20$ & $11.94,1.47$ & 12.55 & $12 \cdot 29,12 \cdot 80$ & $12 \cdot 44$ & $12 \cdot 81,12 \cdot 70$ & $12 \cdot 65$ & $12 \cdot 39,12.91$ & 0.06 & 0.44 & $0.06,0.83$ \\
\hline $\mathrm{DHA}(\%)$ & \multicolumn{2}{|r|}{$<1.97$} & \multicolumn{2}{|c|}{$1.97-2.38$} & \multicolumn{2}{|c|}{$2 \cdot 39,2.86$} & \multicolumn{2}{|r|}{$>2.86$} & & & \\
\hline Model 1 & $12 \cdot 34$ & $12 \cdot 06,12 \cdot 61$ & $12 \cdot 43$ & $12 \cdot 16,12 \cdot 71$ & $12 \cdot 34$ & $12 \cdot 07,12 \cdot 62$ & $12 \cdot 73$ & $12.45,13.00$ & 0.06 & 0.39 & $0.00,0.78$ \\
\hline Model 2 & $12 \cdot 32$ & $12.06,12.59$ & $12 \cdot 43$ & $12 \cdot 18,12 \cdot 69$ & $12 \cdot 40$ & $12 \cdot 14,12 \cdot 66$ & $12 \cdot 68$ & $12.41,12.94$ & 0.08 & 0.35 & $-0.03,0.73$ \\
\hline Model $2+$ hair $\mathrm{Hg}$ & $12 \cdot 32$ & $12.07,12 \cdot 60$ & 12.44 & $12 \cdot 18,12 \cdot 70$ & $12 \cdot 40$ & $12 \cdot 14,12.66$ & 12.66 & $12.40,12.93$ & 0.11 & 0.33 & $-0.06,0.72$ \\
\hline Hair Hg ( $\mu \mathrm{g} / \mathrm{g})$ & \multicolumn{2}{|r|}{$<0.65$} & \multicolumn{2}{|c|}{$0.65-1.30$} & \multicolumn{2}{|c|}{$1.31-2.54$} & \multicolumn{2}{|r|}{$>2.54$} & & & \\
\hline Model 1 & $12 \cdot 31$ & $12 \cdot 03,12.59$ & 12.54 & $12 \cdot 26,12 \cdot 81$ & $12 \cdot 74$ & $12.47,13.02$ & $12 \cdot 24$ & $11.96,12.52$ & 0.40 & -0.07 & $-0.47,0.33$ \\
\hline Model 2 & $12 \cdot 18$ & $11.92,12.45$ & 12.49 & $12 \cdot 23,12 \cdot 75$ & $12 \cdot 74$ & $12.48,13.00$ & $12 \cdot 41$ & $12 \cdot 15,12 \cdot 68$ & 0.13 & 0.23 & $-0.16,0.62$ \\
\hline Model $2+\mathrm{EPA}+\mathrm{DPA}+\mathrm{DHA}$ & $12 \cdot 25$ & $11.99,12.53$ & 12.51 & $12 \cdot 25,12 \cdot 77$ & 12.72 & $12.47,12.98$ & $12 \cdot 34$ & $12.07,12.61$ & 0.89 & 0.08 & $-0.32,0.49$ \\
\hline
\end{tabular}


Table 3. Maximum $\mathrm{VO}_{2}(\mathrm{ml} / \mathrm{min})$ in quartiles of serum long-chain $n-3$ PUFA and hair mercury ${ }^{*}$ (Mean values and $95 \%$ confidence intervals)

\begin{tabular}{|c|c|c|c|c|c|c|c|c|c|c|c|}
\hline & \multicolumn{8}{|c|}{ Exposure quartile } & \multirow[b]{3}{*}{$P_{\text {for trend }}$} & \multirow{2}{*}{\multicolumn{2}{|c|}{ Mean difference $†$}} \\
\hline & \multicolumn{2}{|c|}{1 (n 418) } & \multicolumn{2}{|c|}{2 (n 418) } & \multicolumn{2}{|c|}{3 (n 418) } & \multicolumn{2}{|c|}{$4(n 418)$} & & & \\
\hline & Mean & $95 \% \mathrm{Cl}$ & Mean & $95 \% \mathrm{Cl}$ & Mean & $95 \% \mathrm{Cl}$ & Mean & $95 \% \mathrm{Cl}$ & & Mean & $95 \% \mathrm{Cl}$ \\
\hline$E P A+D P A+D H A(\%)$ & \multicolumn{2}{|c|}{$<3.67$} & \multicolumn{2}{|c|}{$3 \cdot 67-4 \cdot 38$} & \multicolumn{2}{|c|}{$4 \cdot 39-5 \cdot 40$} & \multicolumn{2}{|c|}{$>5.40$} & & & \\
\hline Model $1 \ddagger$ & 2470 & 2418,2522 & 2554 & 2502,2606 & 2581 & 2502,2606 & 2575 & 2523,2627 & 0.01 & 105 & 31,178 \\
\hline Model $2 \S$ & 2483 & 2435,2531 & 2542 & 2496,2589 & 2588 & 2541,2635 & 2566 & 2519,2613 & 0.02 & 83 & 15,152 \\
\hline Model $2+$ hair $\mathrm{Hg}$ & 2490 & 2442,2539 & 2545 & 2498,2592 & 2586 & 2539,2633 & 2557 & 2510,2606 & 0.08 & 67 & $-3,138$ \\
\hline EPA (\%) & \multicolumn{2}{|c|}{$<1.13$} & \multicolumn{2}{|c|}{$1.13-1.49$} & \multicolumn{2}{|c|}{$1.50-2.01$} & \multicolumn{2}{|c|}{$>2.01$} & & & \\
\hline Model 1 & 2492 & 2439,2544 & 2516 & 2464,2568 & 2612 & 2560,2664 & 2560 & 2508,2612 & 0.04 & 68 & $-7,143$ \\
\hline Model 2 & 2495 & 2447,2543 & 2513 & 2466,2559 & 2609 & 2563,2656 & 2563 & 2515,2610 & 0.03 & 68 & $-2,137$ \\
\hline Model $2+$ hair $\mathrm{Hg}$ & 2501 & 2452,2550 & 2517 & 2470,2559 & 2607 & 2561,2654 & 2554 & 2506,2603 & 0.10 & 53 & $-18,124$ \\
\hline DPA (\%) & \multicolumn{2}{|c|}{$<0.48$} & \multicolumn{2}{|c|}{$0.48-0.54$} & \multicolumn{2}{|c|}{$0.55-0.62$} & \multicolumn{2}{|c|}{$>0.62$} & & & \\
\hline Model 1 & 2441 & 2389,2493 & 2546 & 2494,2598 & 2587 & 2535, 2639 & 2606 & 2555,2658 & $<0.001$ & 166 & 92,239 \\
\hline Model 2 & 2476 & 2428,2524 & 2549 & 2502,2595 & 2574 & 2527,2621 & 2581 & 2534,2628 & 0.004 & 105 & 37,173 \\
\hline Model $2+$ hair $\mathrm{Hg}$ & 2481 & 2434,2530 & 2550 & 2503,2596 & 2572 & 2526,2619 & 2576 & 2528,2623 & 0.01 & 94 & 24,163 \\
\hline DHA (\%) & \multicolumn{2}{|c|}{$<1.97$} & \multicolumn{2}{|c|}{$1.97-2.38$} & \multicolumn{2}{|c|}{$2.39-2.86$} & \multicolumn{2}{|c|}{$>2.86$} & & & \\
\hline Model 1 & 2489 & 2437,2541 & 2559 & 2507,2611 & 2535 & 2483,2587 & 2597 & 2544,2649 & 0.01 & 108 & 34,182 \\
\hline Model 2 & 2498 & 2450,2545 & 2557 & 2511,2604 & 2546 & 2499,2592 & 2579 & 2532,2626 & 0.04 & 81 & 13,150 \\
\hline Model $2+$ hair $\mathrm{Hg}$ & 2506 & 2458,2555 & 2559 & 2513,2606 & 2543 & 2496,2590 & 2571 & 2523,2619 & 0.11 & 65 & $-6,135$ \\
\hline Hair Hg $(\mu \mathrm{g} / \mathrm{g})$ & \multicolumn{2}{|c|}{$<0.65$} & \multicolumn{2}{|c|}{$0.65-1.30$} & \multicolumn{2}{|c|}{$1.31-2.54$} & \multicolumn{2}{|c|}{$>2.54$} & & & \\
\hline Model 1 & 2514 & 2461,2567 & 2543 & 2491,2596 & 2590 & 2538,2642 & 2532 & 2479,2585 & 0.84 & 18 & $-59,94$ \\
\hline Model 2 & 2495 & 2447,2542 & 2537 & 2490,2583 & 2584 & 2537,2630 & 2565 & 2516,2613 & 0.09 & 70 & $0.3,140$ \\
\hline Model $2+$ EPA + DPA + DHA & 2507 & 2459,2556 & 2540 & 2493,2587 & 2580 & 2534,2627 & 2552 & 2503,2601 & 0.34 & 44 & $-28,117$ \\
\hline
\end{tabular}

DPA, docosapentaenoic acid

${ }^{*}$ The mean values in the exposure quartiles were analysed using ANCOVA.

† Mean difference between the extreme quartiles.

$\S$ Model 2: adjusted for model $1+\mathrm{BMI}$, current smoking status, leisure-time physical activity, energy intake, carbohydrate intake, alcohol intake, use of drugs for hypertension and C-reactive protein, LDL- and HDL-cholesterol concentrations. 
Table 4. Maximum systolic blood pressure ( $\mathrm{mmHg}$ ) in quartiles of serum long-chain $n-3$ PUFA and hair mercury* (Mean values and $95 \%$ confidence intervals)

\begin{tabular}{|c|c|c|c|c|c|c|c|c|c|c|c|}
\hline & \multicolumn{8}{|c|}{ Exposure quartile } & \multirow[b]{3}{*}{$P_{\text {for trend }}$} & \multirow{2}{*}{\multicolumn{2}{|c|}{ Mean difference $\dagger$}} \\
\hline & \multicolumn{2}{|c|}{$1(n 418)$} & \multicolumn{2}{|c|}{$2(n 418)$} & \multicolumn{2}{|c|}{$3(n 418)$} & \multicolumn{2}{|c|}{$4(n 418)$} & & & \\
\hline & Mean & $95 \% \mathrm{Cl}$ & Mean & $95 \% \mathrm{Cl}$ & Mean & $95 \% \mathrm{Cl}$ & Mean & $95 \% \mathrm{Cl}$ & & Mean & $95 \% \mathrm{Cl}$ \\
\hline $\mathrm{EPA}+\mathrm{DPA}+\mathrm{DHA}(\%)$ & \multicolumn{2}{|r|}{$<3.67$} & \multicolumn{2}{|c|}{$3.67-4.38$} & \multicolumn{2}{|c|}{$4 \cdot 38-5 \cdot 40$} & \multicolumn{2}{|r|}{$>5.40$} & & & \\
\hline Model $1 \ddagger$ & 204.9 & $202 \cdot 3,207 \cdot 4$ & 206.6 & $204 \cdot 1,209 \cdot 2$ & $207 \cdot 0$ & $204.5,209 \cdot 6$ & 207.8 & $205 \cdot 2,210 \cdot 3$ & 0.14 & $2 \cdot 9$ & $-0.7,6.5$ \\
\hline Model $2 \S$ & $206 \cdot 1$ & $203 \cdot 6,208 \cdot 7$ & $206 \cdot 7$ & $204 \cdot 2,209 \cdot 2$ & $206 \cdot 5$ & $203 \cdot 9,209 \cdot 0$ & $207 \cdot 0$ & $204.5,209 \cdot 5$ & 0.69 & 0.9 & $-2 \cdot 8,4.5$ \\
\hline Model $2+$ hair $\mathrm{Hg}$ & $206 \cdot 6$ & $204 \cdot 0,209 \cdot 2$ & $206 \cdot 9$ & $204 \cdot 4,209 \cdot 4$ & $206 \cdot 3$ & $203 \cdot 8,208 \cdot 8$ & 206.5 & $204 \cdot 0,209 \cdot 1$ & 0.93 & -0.03 & $-3.8,3.8$ \\
\hline EPA (\%) & \multicolumn{2}{|r|}{$<1.13$} & \multicolumn{2}{|c|}{$1.13-1.49$} & \multicolumn{2}{|c|}{$1.49-2.01$} & \multicolumn{2}{|r|}{$>2.01$} & & & \\
\hline Model 1 & $206 \cdot 4$ & $203.8,208.9$ & 205.0 & $202 \cdot 4,207 \cdot 5$ & 207.1 & $204 \cdot 6,209 \cdot 7$ & $207 \cdot 9$ & $205 \cdot 3,210 \cdot 4$ & 0.22 & 1.5 & $-2 \cdot 2,5 \cdot 2$ \\
\hline Model 2 & 207.5 & $205 \cdot 0,210 \cdot 1$ & $205 \cdot 3$ & $202 \cdot 8,207 \cdot 8$ & $206 \cdot 7$ & $204 \cdot 2,209 \cdot 2$ & 206.7 & $204 \cdot 2,209 \cdot 3$ & 0.98 & -0.8 & $-4 \cdot 6,2 \cdot 9$ \\
\hline Model $2+$ hair $\mathrm{Hg}$ & 207.9 & $205 \cdot 3,210 \cdot 5$ & 205.6 & $203 \cdot 1,208 \cdot 1$ & $206 \cdot 6$ & $204 \cdot 1,209 \cdot 1$ & $206 \cdot 3$ & $203 \cdot 7,208 \cdot 9$ & 0.63 & -1.6 & $-5 \cdot 5,2 \cdot 2$ \\
\hline DPA (\%) & \multicolumn{2}{|r|}{$<0.48$} & \multicolumn{2}{|c|}{$0.48-0.54$} & \multicolumn{2}{|c|}{$0.54-0.62$} & \multicolumn{2}{|r|}{$>0.62$} & & & \\
\hline Model 1 & $205 \cdot 3$ & $202 \cdot 8,207.9$ & 205.7 & $203 \cdot 2,208 \cdot 3$ & 209.1 & $206 \cdot 6,211 \cdot 7$ & $206 \cdot 2$ & $203 \cdot 6,208 \cdot 7$ & 0.38 & 0.9 & $-2 \cdot 7,4.5$ \\
\hline Model 2 & $205 \cdot 2$ & $202 \cdot 7,207 \cdot 8$ & 205.4 & $202 \cdot 9,207 \cdot 9$ & 209.1 & $206 \cdot 6,211 \cdot 6$ & $206 \cdot 6$ & $204 \cdot 1,209 \cdot 1$ & 0.25 & 1.3 & $-2 \cdot 3,5 \cdot 0$ \\
\hline Model $2+$ hair $\mathrm{Hg}$ & $205 \cdot 5$ & $202 \cdot 9,208 \cdot 1$ & $205 \cdot 4$ & $202 \cdot 9,207 \cdot 9$ & 209.1 & $206 \cdot 6,211 \cdot 6$ & $206 \cdot 3$ & $203 \cdot 8,208 \cdot 8$ & 0.40 & 0.8 & $-2.9,4.5$ \\
\hline DHA (\%) & \multicolumn{2}{|r|}{$<1.97$} & \multicolumn{2}{|c|}{$1.97-2.38$} & \multicolumn{2}{|c|}{$2.38-2.86$} & \multicolumn{2}{|r|}{$>2.86$} & & & \\
\hline Model 1 & $203 \cdot 7$ & $201 \cdot 1,206 \cdot 2$ & 208.0 & $205 \cdot 5,210 \cdot 6$ & 207.5 & $204 \cdot 9,210 \cdot 0$ & $207 \cdot 1$ & $204 \cdot 6,209 \cdot 7$ & 0.11 & 3.5 & $-0.1,7 \cdot 1$ \\
\hline Model 2 & 204.8 & $202 \cdot 3,207 \cdot 4$ & 207.9 & $205 \cdot 4,210 \cdot 4$ & $207 \cdot 2$ & $204 \cdot 7,209 \cdot 7$ & $206 \cdot 4$ & $203.8,208.9$ & 0.56 & 1.6 & $-2 \cdot 1,5 \cdot 3$ \\
\hline Model $2+$ hair $\mathrm{Hg}$ & $205 \cdot 2$ & $202 \cdot 6,207 \cdot 8$ & 208.0 & $205.5,210.5$ & 207.0 & $204.5,209.5$ & $206 \cdot 0$ & $203 \cdot 4,208 \cdot 6$ & 0.90 & 0.8 & $-3 \cdot 0,4 \cdot 6$ \\
\hline Hair $\mathrm{Hg}(\mu \mathrm{g} / \mathrm{g})$ & \multicolumn{2}{|r|}{$<0.65$} & \multicolumn{2}{|c|}{$0.65-1.30$} & \multicolumn{2}{|c|}{$1.30-2.54$} & \multicolumn{2}{|r|}{$>2.54$} & & & \\
\hline Model 1 & $206 \cdot 3$ & $203 \cdot 7,208 \cdot 8$ & 204.9 & $202 \cdot 3,207 \cdot 5$ & $205 \cdot 8$ & $203 \cdot 3,208 \cdot 4$ & $209 \cdot 3$ & $206 \cdot 7,211.9$ & 0.03 & 3.1 & $-0.6,6 \cdot 8$ \\
\hline Model 2 & $207 \cdot 0$ & $204.4,209.5$ & 205.1 & $202 \cdot 6,207 \cdot 6$ & $205 \cdot 3$ & $202 \cdot 8,207 \cdot 8$ & 209.0 & $206 \cdot 4,211 \cdot 6$ & 0.11 & $2 \cdot 0$ & $-1 \cdot 7,5 \cdot 8$ \\
\hline Model $2+E P A+D P A+D H A$ & 206.9 & $204 \cdot 3,209 \cdot 5$ & $205 \cdot 1$ & $202 \cdot 5,207 \cdot 6$ & $205 \cdot 3$ & $202 \cdot 8,207 \cdot 8$ & 209.1 & $206 \cdot 4,211 \cdot 7$ & $0 \cdot 10$ & $2 \cdot 2$ & $-1 \cdot 7,6 \cdot 1$ \\
\hline
\end{tabular}


the median of $1.30 \mu \mathrm{g} / \mathrm{g}$, although the $P_{\text {for }}$ interaction was statistically significant only for total $n-3$ PUFA $(P=0.03)$ and EPA $(P=0.02)$, but not for DPA $(P=0 \cdot 14)$ or DHA $(P=0 \cdot 18)$ (online Supplementary Table S2).

\section{Serum long-chain n-3 PUFA, hair mercury and maximal systolic blood pressure during exercise}

The mean maximal SBP during exercise was $206 \cdot 6$ (SD 26.5) $\mathrm{mmHg}$. Serum long-chain $n$-3 PUFA were not associated with maximal SBP during exercise (Table 4). Although hair $\mathrm{Hg}$ was associated with a trend towards higher maximal SBP after adjustment for age and examination year, further adjustments attenuated the association and it was no longer statistically significant (Table 4). Hair Hg did not modify the associations between the long-chain $n-3$ PUFA and maximal SBP during exercise as well $\left(P_{\text {for interaction }}=0.23\right.$ for total long-chain $n$-3 PUFA, $P=0.39$ for EPA, $P=0.13$ for DPA and $P=0.26$ for DHA) (online Supplementary Table S3).

\section{Sensitivity analyses}

The associations were generally similar when we excluded participants using hypertension medication ( $n$ 209) from the analyses. For example, the mean ECP in quartiles of serum total long-chain $n-3$ PUFA after excluding those using hypertension medication was $12.20,12.61,12.84$ and $12.62 \mathrm{ml} / \mathrm{mmHg}$ (model 2, $P_{\text {trend }}=0.05$ ), the mean $\mathrm{VO}_{2 \max } 2505,2582,2638$ and $2585 \mathrm{ml} / \mathrm{min}\left(P_{\text {trend }}=0.05\right)$ and the mean maximal SBP during exercise 207.1, 207.6, 207.1 and $207.7 \mathrm{mmHg}\left(P_{\text {trend }}=0.83\right)$ (other data not shown).

\section{Discussion}

In this cross-sectional study among 1627 middle-aged and older men from eastern Finland, the serum long-chain $n-3$ PUFA were associated with higher ECP and $\mathrm{VO}_{2 \max }$, but not with maximal SBP during exercise. However, the clinical significance of the associations was quite modest, but this can be expected because exercise capacity is to a large extent determined by genetics and physical activity ${ }^{(32-34)}$. Furthermore, although hair $\mathrm{Hg}$ concentration was not associated with ECP, higher hair $\mathrm{Hg}$ concentration modestly attenuated the associations of the long-chain $n$-3 PUFA with $\mathrm{VO}_{2 \max }$ and ECP.

There is little evidence regarding the association between long-chain $n$-3 PUFA and ECP and its components; three small, randomised trials found that $\mathrm{VO}_{2 \max }$ was increased dose-dependently by fish oil supplementation ${ }^{(12,18,20)}$, but this has not been observed in all supplementation studies ${ }^{(13-17,19)}$. Moreover, in one study, a DHA-rich meal led to lower systemic vascular resistance and to a smaller increase in SBP during exercise compared with a control meal ${ }^{(21)}$, whereas two other studies did not find any effect of fish oil supplementation on exercise-induced blood pressure ${ }^{(19,22)}$. It has been reported that intake of long-chain $n$-3 PUFA is associated with lower resting blood pressure ${ }^{(35)}$. We have previously reported a modest, inverse association between long-chain $n$-3 PUFA and resting SBP in the 11-year examination of the KIHD cohort ${ }^{(36,37)}$. However, in the current study, we could not find such an association. The lack of association might be due to haemodynamic response to exercise, which is not taken into account for SBP at rest ${ }^{(38)}$.

A possible mechanism underlying the beneficial impact of the serum long-chain $n-3$ PUFA on exercise capacity during an exercise test might be explained by the effect of the long-chain $n-3$ PUFA on the vascular endothelial functions ${ }^{(39)}$, such as improvement in vascular reactivity ${ }^{(40,41)}$, increased production of endogenous antioxidant enzymes and decreased inflammatory cytokines $^{(40,41)}$ and bioavailability of endothelial nitric oxide ${ }^{(40,42)}$.

We have previously found that higher hair $\mathrm{Hg}$ concentration attenuated the inverse associations of the long-chain $n$-3 PUFA with CVD outcomes in the KIHD cohort ${ }^{(10,36)}$. In the current study, hair $\mathrm{Hg}$ attenuated the associations of the long-chain $n-3$ PUFA with $\mathrm{VO}_{2 \max }$ and ECP, although the interaction was statistically significant only for $\mathrm{VO}_{2 \max }$. This attenuation may be at least partially explained by the role of $\mathrm{Hg}$ on endothelial dysfunction by reduction in nitric oxide bioavailability and nitric oxide synthase expression ${ }^{(43)}$

The strengths of our study include the use of serum longchain $n$-3 PUFA and hair $\mathrm{Hg}$ as exposures instead of dietary intakes. As serum fatty acids and hair $\mathrm{Hg}$ are objective biomarkers for exposure ${ }^{(23,44)}$, their use reduced the bias by misclassification, which would attenuate the associations towards the null. Other strengths include the extensive examination of potential confounders and the large number of participants with the assessment of $\mathrm{VO}_{2 \max }$, which is considered to be the 'gold standard' for measuring cardiorespiratory fitness $^{(6)}$. A limitation of this study is that it is based on an ethnically homogenic population of middle-aged and older men, which may limit the generalisability of our results. In addition, the average hair $\mathrm{Hg}$ concentrations are somewhat higher in the KIHD cohort compared with other study populations that have reported $\mathrm{Hg}$ exposure ${ }^{(45,46)}$. Therefore, our results may not be generalisable to study populations with lower average $\mathrm{Hg}$ exposure.

In conclusion, our results suggest that higher circulating concentrations of long-chain $n-3$ PUFA, mainly a marker of fish consumption in this study population, are associated with higher ECP and $\mathrm{VO}_{2 \max }$ in middle-aged and older men from eastern Finland. As low cardiorespiratory fitness $\left(\mathrm{VO}_{2 \max }\right)$ and low ECP are risk factors for $\mathrm{CVD}^{(3-6,8)}$, these results could partially explain how long-chain $n$-3 PUFA may reduce the risk of cardiac mortality.

\section{Acknowledgements}

The authors thank the staff of the Kuopio Research Institute of Exercise Medicine and the Research Institute of Public Health, University of Eastern Finland, for data collection.

The study was supported by the University of Eastern Finland. This research received no specific grant from any funding agency or from commercial or not-for-profit sectors.

The authors' contributions were as follows: B. T., S. K., T.-P. T. and J. K. V. contributed to the conception and design of the research; S. K., and T.-P. T. acquired the data; B. T. and J. K. V. analysed the data and interpreted the results; B. T. drafted the 
manuscript; and all the authors critically revised the paper and approved the final version of the manuscript.

There are no conflicts of interest.

\section{Supplementary material}

For supplementary material/s referred to in this article, please visit http://dx.doi.org/doi:10.1017/S0007114516002142

\section{References}

1. Kokkinos P, Myers J, Kokkinos JP, et al. (2008) Exercise capacity and mortality in black and white men. Circulation 117, 614-622.

2. Morris CK, Ueshima K, Kawaguchi T, et al. (1991) The prognostic value of exercise capacity: a review of the literature. Am Heart J 122, 1423-1431.

3. Kurl S, Laukkanen JA, Niskanen L, et al. (2005) Cardiac power during exercise and the risk of stroke in men. Stroke 36, 820-824.

4. Kurl S, Jae SY, Kauhanen J, et al. (2015) Exercise cardiac power and the risk of sudden cardiac death in a long-term prospective study. Int J Cardiol 181, 155-159.

5. Laukkanen JA, Lakka TA, Rauramaa R, et al. (2001) Cardiovascular fitness as a predictor of mortality in men. Arch Intern Med 161, 825-831.

6. Laukkanen JA, Kurl S \& Salonen JT (2002) Cardiorespiratory fitness and physical activity as risk predictors of future atherosclerotic cardiovascular diseases. Curr Atheroscler Rep 4, 468-476.

7. Kurl S, Laukkanen JA, Rauramaa R, et al. (2001) Systolic blood pressure response to exercise stress test and risk of stroke. Stroke 32, 2036-2041.

8. Laukkanen JA, Mäkikallio TH, Rauramaa R, et al. (2010) Cardiorespiratory fitness is related to the risk of sudden cardiac death: a population-based follow-up study. J Am Coll Cardiol 56, 1476-1483.

9. Mozaffarian D \& Wu JH (2011) Omega-3 fatty acids and cardiovascular disease: effects on risk factors, molecular pathways, and clinical events. J Am Coll Cardiol 58, 2047-2067.

10. Virtanen JK, Voutilainen S, Rissanen TH, et al. (2005) Mercury, fish oils, and risk of acute coronary events and cardiovascular disease, coronary heart disease, and all-cause mortality in men in eastern Finland. Arterioscler Thromb Vasc Biol 25, 228-233.

11. Virtanen JK, Laukkanen JA, Mursu J, et al. (2012) Serum long-chain $n-3$ polyunsaturated fatty acids, mercury, and risk of sudden cardiac death in men: a prospective populationbased study. PLOS ONE 7, e41046.

12. Guezennec CY, Nadaud JF, Satabin P, et al. (1989) Influence of polyunsaturated fatty acid diet on the hemorrheological response to physical exercise in hypoxia. Int J Sports Med 10, 286-291.

13. Boss A, Lecoultre V, Ruffieux C, et al. (2010) Combined effects of endurance training and dietary unsaturated fatty acids on physical performance, fat oxidation and insulin sensitivity. Br J Nutr 103, 1151-1159.

14. Raastad T, Hastmark A \& Strømme S (1997) Omega-3 fatty acid supplementation does not improve maximal aerobic power, anaerobic threshold and running performance in well-trained soccer players. Scand J Med Sci Sports 7, 25-31.

15. Brilla LR \& Landerholm TE (1990) Effect of fish oil supplementation and exercise on serum lipids and aerobic fitness. J Sports Med Phys Fitness 30, 173-180.
16. Oostenbrug GS, Mensink RP, Hardeman MR, et al. (1997) Exercise performance, red blood cell deformability, and lipid peroxidation: effects of fish oil and vitamin E. J Appl Physiol (1985) 83, 746-752.

17. Kawabata F, Neya M, Hamazaki K, et al. (2014) Supplementation with eicosapentaenoic acid-rich fish oil improves exercise economy and reduces perceived exertion during submaximal steady-state exercise in normal healthy untrained men. Biosci Biotechnol Biochem 78, 2081-2088.

18. Żebrowska A, Mizia-Stec K, Mizia M, et al. (2015) Omega-3 fatty acids supplementation improves endothelial function and maximal oxygen uptake in endurance-trained athletes. Eur J Sport Sci 15, 305-314.

19. Peoples GE, McLennan PL, Howe PR, et al. (2008) Fish oil reduces heart rate and oxygen consumption during exercise. J Cardiovasc Pharmacol 52, 540-547.

20. Leaf D \& Rauch C (1988) Omega-3 supplementation and estimated VO2 max: a double blind randomized controlled trial in athletes. Ann Sport Med 4, 37-40.

21. Rontoyanni VG, Hall WL, Pombo-Rodrigues S, et al. (2012) A comparison of the changes in cardiac output and systemic vascular resistance during exercise following high-fat meals containing DHA or EPA. Br J Nutr 108, 492-499.

22. O'Keefe JH, Abuissa H, Sastre A, et al. (2006) Effects of omega-3 fatty acids on resting heart rate, heart rate recovery after exercise, and heart rate variability in men with healed myocardial infarctions and depressed ejection fractions. Am J Cardiol 97, 1127-1130.

23. Roman HA, Walsh TL, Coull BA, et al. (2011) Evaluation of the cardiovascular effects of methylmercury exposures: current evidence supports development of a dose-response function for regulatory benefits analysis. Environ Health Perspect 119, 607-614.

24. Salonen JT (1988) Is there a continuing need for longitudinal epidemiologic research? The Kuopio Ischaemic Heart Disease Risk Factor Study. Ann Clin Res 20, 46-50.

25. Salonen JT, Nyyssonen K, Korpela H, et al. (1992) High stored iron levels are associated with excess risk of myocardial infarction in eastern Finnish men. Circulation 86, 803-811.

26. Lakka TA, Venalainen JM, Rauramaa R, et al. (1994) Relation of leisure-time physical activity and cardiorespiratory fitness to the risk of acute myocardial infarction in men. $N$ Engl J Med 330, 1549-1554.

27. Voutilainen S, Rissanen TH, Virtanen J, et al. (2001) Low dietary folate intake is associated with an excess incidence of acute coronary events: the Kuopio Ischemic Heart Disease Risk Factor Study. Circulation 103, 2674-2680.

28. Laaksonen D, Lakka T, Lakka H, et al. (2002) Serum fatty acid composition predicts development of impaired fasting glycaemia and diabetes in middle-aged men. Diabet Med 19, 456-464.

29. Salonen JT, Seppanen K, Nyyssonen K, et al. (1995) Intake of mercury from fish, lipid peroxidation, and the risk of myocardial infarction and coronary, cardiovascular, and any death in eastern Finnish men. Circulation 91, 645-655.

30. Lakka TA, Laukkanen JA, Rauramaa R, et al. (2001) Cardiorespiratory fitness and the progression of carotid atherosclerosis in middle-aged men. Ann Intern Med 134, 12-20.

31. Ferguson CJ (2009) An effect size primer: a guide for clinicians and researchers. Prof Psychol Res Pract 40, 532-538.

32. Bouchard C, Boulay MR, Simoneau J, et al. (1988) Heredity and trainability of aerobic and anaerobic performances. An update. Sports Med 5, 69-73.

33. Rankinen T, Perusse L, Rauramaa R, et al. (2001) The human gene map for performance and health-related fitness phenotypes. Med Sci Sports Exerc 33, 855-867. 
34. Dionne FT, Turcotte L, Thibault MC, et al. (1991) Mitochondrial DNA sequence polymorphism, VO2max, and response to endurance training. Med Sci Sports Exerc 23, 177-185.

35. Miller PE, Van Elswyk M \& Alexander DD (2014) Long-chain omega-3 fatty acids eicosapentaenoic acid and docosahexaenoic acid and blood pressure: a meta-analysis of randomized controlled trials. Am J Hypertens 27, 885-896.

36. Virtanen JK, Nyantika AN, Kauhanen J, et al. (2012) Serum long-chain $n$-3 polyunsaturated fatty acids, methylmercury and blood pressure in an older population. Hypertens Res $\mathbf{3 5}$, 1000-1004.

37. Nyantika A, Tuomainen T, Kauhanen J, et al. (2014) Serum long-chain omega-3 polyunsaturated fatty acids and future blood pressure in an ageing population. J Nutr Health Aging 19, 498-503.

38. McHam SA, Marwick TH, Pashkow FJ, et al. (1999) Delayed systolic blood pressure recovery after graded exercise: an independent correlate of angiographic coronary disease. J Am Coll Cardiol 34, 754-759.

39. Khan F, Elherik K, Bolton-Smith C, et al. (2003) The effects of dietary fatty acid supplementation on endothelial function and vascular tone in healthy subjects. Cardiovasc Res 59, 955-962.
40. Wu G \& Meininger CJ (2002) Regulation of nitric oxide synthesis by dietary factors. Annu Rev Nutr 22, 61-86.

41. Mickleborough TD (2013) Omega-3 polyunsaturated fatty acids in physical performance optimization. Int J Sport Nutr Exerc Metab 23, 83-96.

42. Juturu V (2008) Omega-3 fatty acids and the cardiometabolic syndrome. J Cardiometab Syndr 3, 244-253.

43. Furieri LB, Galán M, Avendaño MS, et al. (2011) Endothelial dysfunction of rat coronary arteries after exposure to low concentrations of mercury is dependent on reactive oxygen species. Br J Pharmacol 162, 1819-1831.

44. Hodson L, Skeaff CM \& Fielding BA (2008) Fatty acid composition of adipose tissue and blood in humans and its use as a biomarker of dietary intake. Prog Lipid Res $\mathbf{4 7}$, 348-380.

45. Wennberg M, Bergdahl IA, Hallmans G, et al. (2011) Fish consumption and myocardial infarction: a second prospective biomarker study from northern Sweden. Am J Clin Nutr 93, 27-36.

46. Mozaffarian D, Shi P, Morris JS, et al. (2011) Mercury exposure and risk of cardiovascular disease in two US cohorts. $N$ Engl $J$ Med 364, 1116-1125. 